\title{
IDENTIFICATION OF NEUROPEPTIDE Y \\ IN SUPERIOR CERVICAL GANGLION NEURONS THAT PROJECT TO THE OESOPHAGUS - A COMBINED IMMUNOHISTOCHEMICAL LABELLING AND RETROGRADE TRACING STUDY IN PIGS
}

\author{
Liliana RYTEL ${ }^{1}$, Anna SNARSKA ${ }^{1}$, Slawomir GONKOWSKI ${ }^{2}$, Joanna WOJTKIEWICZ ${ }^{3}$, \\ Ottó SzENCI ${ }^{4}$ and Przemyslaw SOBIECH ${ }^{1 *}$ \\ ${ }^{1}$ Division of Internal Disease with Clinic, University of Warmia and Mazury in Olsztyn, \\ Oczapowskiego Str. 13, 10-719 Olsztyn, Poland; ${ }^{2}$ Division of Clinical Physiology, \\ University of Warmia and Mazury in Olsztyn, Poland; ${ }^{3}$ Department of Pathophysiology, \\ School of Medicine, Collegium Medicum, University of Warmia and Mazury in Olsztyn, \\ Olsztyn, Poland; ${ }^{4}$ MTA-SZIE Large Animal Clinical Research Group, Üllő, Hungary
}

(Received 4 July 2018; accepted 20 November 2018)

\begin{abstract}
Neuropeptide Y (NPY) is a neuronal active substance taking part in the regulation of gastrointestinal (GI) tract activity. This study used retrograde neuronal tracing and immunofluorescence methods to analyse NPY-positive neurons located in superior cervical ganglion and supplying the cervical oesophagus in the pig. The presence of NPY was observed in $30 \%$ of all neurons supplying the part of oesophagus studied. Probably the number of Fast Blue (FB) positive cells depends on the area of the wall injected with FB and the fragment of oesophagus studied. Therefore, the obtained results indicate that the described peptide is an important factor in the extrinsic innervation of this part of the GI tract.
\end{abstract} peptide $\mathrm{Y}$

Key words: Oesophagus, extrinsic innervation, retrograde tracing, neuro-

The innervation of the gastrointestinal (GI) tract consists of two parts: the enteric nervous system (ENS) (Szymanska et al., 2018) and extrinsic innervation (Majewski et al., 2002; Rytel and Calka, 2016). The ENS, composed of neuronal cells grouped into intramural ganglia and plexuses, is localised in the wall of the GI tract. Since the ENS shows significant independence from the central nervous system, it is often called 'the second brain' (Grubišić et al., 2018). Nevertheless, the ENS is under the control of the central nervous system by the extrinsic innervation of the GI tract. Neurons supplying the GI tract are located in various sympathetic, parasympathetic and sensory ganglia, and their exact localisation depends on the innervated gastrointestinal fragment. The majority of studies on the extrinsic innervation of the stomach and intestine have been based on the retro-

\footnotetext{
*Corresponding author; E-mail: psobiech@uwm.edu.pl; Phone: 0048 (89) 523-3440
} 
grade tracing technique, which consists of the injection of a retrograde tracer (a substance that may be transported from nerve endings to the perikaryon) into the wall of the GI tract (Bedwell and Tinsley, 2018). Among a wide range of substances showing the features of retrograde tracers, fast blue (FB) is the most commonly used because it is effectively transported retrogradely over long distances in various animal models and is characterised by a long degradation time in the nervous tissue (Sarnat, 2015). Moreover, neurons labelled with FB are clearly visible, and the results obtained during studies using FB are reproducible (Zalecki et al., 2012).

It is known that the oesophagus may receive innervation from jugularnodose, sphenopalatine, stellate and dorsal root ganglia (Yu et al., 2015), but the major adrenergic innervation of this part of the GI tract derives from the superior cervical ganglia (Wojtkiewicz et al., 2011; Juranek and Wojtkiewicz, 2015). To date, a wide range of active substances have been noted both in the ENS and extrinsic innervation (Majewski et al., 2002; Obremski et al., 2015; Rytel and Calka, 2016; Szymanska et al., 2018), including neuropeptide Y (NPY).

It is known that NPY in the GI tract is an inhibitory factor, which may participate in the regulation of various gastrointestinal functions, including motility and secretory activity of the intestine (Forbes and Cox, 2014; Louzao et al., 2015). Moreover, NPY affects the mesenteric blood flow by the contraction of vascular muscles and takes part in the pathological processes within the GI tract (Malmström, 2000; De Fontgalland et al., 2008; Chandrasekharan et al., 2008). Contrary to the ENS, where the presence of NPY has been described in various mammalian species including humans (Wang et al., 2006), knowledge concerning the participation of this substance in extrinsic innervation of the GI tract is very scarce (Konturek et al., 2004). On the other hand, previous studies have described NPY as an important neuronal factor occurring in neuronal cells located in the superior cervical ganglion (SCG; Landry et al., 2000; Wojtkiewicz et al., 2011; Masliukov et al., 2012).

The aim of the present study was to investigate, for the first time, the presence of NPY in SCG neurons supplying the cervical part of the oesophagus in the domestic pig which, due to its neurochemical, physiological and anatomical similarity to humans, seems to be an optimal animal model for studies of the GI tract (Oltean et al., 2017).

\section{Materials and methods}

The present study was performed on five juvenile female pigs of the Large White Polish breed (body weight: $12-18 \mathrm{~kg}$ ), which were kept under standard laboratory conditions. All procedures followed the instructions of the Local Ethics Committee of the University of Warmia and Mazury in Olsztyn (Resolution No. 47/2006). The experiment was also performed in accordance with the guidelines 
set forth by Directive 2010/63/EU of the European Parliament and of the Council on the protection of animals used for scientific purposes.

After a five-day adaptation period, the pigs were subjected to premedication with $0.4 \mathrm{mg} / \mathrm{kg}$ atropine s.c. (Polfa, Poland,) and $0.8 \mathrm{mg} / \mathrm{kg}$ propionyl promazine i.m. (Stresnil, Janssen, Belgium) and after about $20 \mathrm{~min}$ general anaesthesia was induced using $20 \mathrm{mg} / \mathrm{kg}$ sodium thiopental i.v. (Thiopental; Sandoz, Austria). The left side of the wall of the cervical oesophagus (at the level of the lower edge of the larynx and in an area of $2 \mathrm{~cm}^{2}$ ) was injected with $30 \mu \mathrm{L}$ of a $5 \%$ aqueous solution of fast blue (FB, Dr. K. Illing GmbH \& KG, Germany; four injections, $5 \mu \mathrm{L}$ each) using a Hamilton syringe equipped with a 26-gauge needle. Great attention was paid to avoiding any contamination of the surrounding tissues with FB due to the hydrostatic leakage from the injection canal. After four weeks, the pigs were subjected to general anaesthesia (using the method described above) and euthanised by an overdose of sodium thiopental (Thiopental, Sandoz, Kundl-Rakusko, Austria; $10 \mathrm{mg} / \mathrm{kg}$ of body weight, i.v.). Afterwards, they were perfused transcardially with $4 \%$ buffered paraformaldehyde (pH 7.4) and the left superior cervical ganglia were collected from all animals.

Table 1

Description of primary and secondary antibodies used for immunohistochemistry

\begin{tabular}{|c|c|c|c|c|}
\hline \multicolumn{5}{|c|}{ Primary antibodies } \\
\hline Antisera & Code & Host species & Dilution & Supplier \\
\hline TH & MAB 318 & Mouse & $1: 80$ & Chemicon Int. Inc., UK \\
\hline NPY & NA 1233 & Rabbit & $1: 10,000$ & Biomol Res. Lab. Inc., US \\
\hline \multicolumn{5}{|c|}{ Secondary antibodies } \\
\hline Reagent & & & Dilution & Supplier \\
\hline \multicolumn{3}{|c|}{$\begin{array}{l}\text { Donkey anti-mouse IgG }(\mathrm{H}+\mathrm{L}) \text { conjugated } \\
\text { with FITC }\end{array}$} & $1: 800$ & 715-095-151; Jackson IR Lab, US; \\
\hline \multicolumn{3}{|c|}{$\begin{array}{l}\left.\text { CY3-conjugated } \mathrm{F}(\mathrm{ab})^{\prime}\right)_{2} \text { donkey anti-rabbit } \\
\operatorname{IgG}(\mathrm{H}+\mathrm{L})\end{array}$} & $1: 9,000$ & 711-166-152, Jackson IR Lab, US; \\
\hline
\end{tabular}

Tissues were rinsed in a $0.1 \mathrm{M}$ buffer solution at $\mathrm{pH} 7.4$ for $72 \mathrm{~h}$, then put into $18 \%$ sucrose solution and kept at $4{ }^{\circ} \mathrm{C}$. After at least three weeks, the ganglia were frozen at $-22{ }^{\circ} \mathrm{C}$, cut with a cryostat into $10-\mu \mathrm{m}$ serial sections and mounted on microscopic slides. Sections were examined under an Olympus BX51 fluorescence microscope (Olympus Life Sciences) to localise all FB-positive neurons. Strips of the SCG with neurons labelled with FB (at least 100 FB-labelled neurons from each animal) were subjected to the routine double immunofluorescence technique previously described by Gonkowski et al. (2009) (Table 1). 
Briefly, this method included the following steps: (1) drying the slides with tissues for $1 \mathrm{~h}$ at room temperature (RT); (2) incubation with a blocking solution ( $10 \%$ normal goat serum, $0.1 \%$ bovine serum albumin, $0.01 \% \mathrm{NaN}_{3}$, Triton $\mathrm{X}$ 100 and thimerosal in PBS) for $1 \mathrm{~h}$; (3) incubation with the mixture of two primary antisera obtained from various species and directed towards tyrosine hydroxylase $(\mathrm{TH})$ and neuropeptide Y. Incubation was performed in humid chamber (overnight); (4) incubation with the secondary antibodies conjugated with FITC and CY3 to visualise the complex of primary antisera bound to appropriate antigens ( $1 \mathrm{~h}$ at RT). Standard controls to confirm the specificity of the method, i.e. pre-absorption of the primary antibody with appropriate antigen and omission and replacement of primary antibody with non-immune serum, were performed. During the pre-absorption test, sections of SCG were incubated with a 'working' dilution of anti-NPY or anti-TH antibodies, which were pre-absorbed for $18 \mathrm{~h}$ at $37^{\circ} \mathrm{C}$ with $20 \mu \mathrm{g}$ of human NPY or TH, respectively.

Labelled sections of the SCG were evaluated under an Olympus BX51 microscope equipped with appropriate filters for FITC (fluorescent filter set for FITC fluorescein with an emission wavelength of 513-556 nm and an excitation wavelength of 467-498 nm), FB (UV-2A filter set with an excitation wavelength of 330-380 nm and a cut-on wavelength of $400 \mathrm{~nm}$ ) and CY3 (fluorescence filter set for CY3 fluorescence dye with an emission wavelength of 570-613 nm and an excitation wavelength of 513-556 nm) to determine the percentage of neurons immunoreactive to NPY with respect to the overall number of FB-positive cells. Finally, the obtained data were pooled and expressed as mean \pm SEM.

\section{Results}

FB-labelled neurons were noted in various parts of the SCG (Table 2).

Table 2

Percentages of nerve cells immunoreactive to particular neuronal factors in porcine superior cervical ganglion neurons that project to the oesophagus

\begin{tabular}{lllll}
\hline Animal no. & $\mathrm{TH}^{+} / \mathrm{NPY}^{+}$ & $\mathrm{TH}^{+} / \mathrm{NPY}^{-}$ & $\mathrm{TH}^{-} / \mathrm{NPY}^{+}$ & $\mathrm{TH}^{-} / \mathrm{NPY}^{-}$ \\
\hline 1 & 21.1 & 39.5 & 8.4 & 34.7 \\
2 & 33.5 & 36.2 & 1.8 & 38.5 \\
3 & 22.2 & 34.8 & 6.7 & 35.4 \\
4 & 30.9 & 24.1 & 2.8 & 39.6 \\
5 & 25.5 & 18.7 & 1.3 & 38.7 \\
\hline Mean \pm SEM & $26.6 \pm 5.4$ & $30.7 \pm 8.8$ & $4.2 \pm 3.2$ & $37.4 \pm 2.2$ \\
\hline
\end{tabular}

$\mathrm{TH}=$ tyrosine hydroxylase; NPY = neuropeptide $\mathrm{Y} ; \mathrm{SEM}=$ standard error of the mean 
Most often these neurons occurred individually or in groups of two or three neurons (Fig. 1). The presence of TH was observed in 56.6\% of SCG neurons supplying the cervical part of the oesophagus. The percentage of FBlabelled neurons simultaneously immunoreactive to NPY was considerably lower and amounted to $30 \%$. Moreover, during a study on co-localisation of $\mathrm{TH}$ and NPY it was found that among neurons labelled with FB, $26.6 \pm 5.4 \%$ simultaneously showed the presence of TH and NPY, $30.7 \pm 8.8 \%$ were immunoreactive to TH but not to NPY, and only $4.2 \pm 3.2 \%$ were neuronal cells showing the presence of NPY although TH was negative. During the present study, no differences were observed between individual animals in the immunoreactivity of FBpositive cells to the substances studied.

\section{Discussion}

The present study confirms the results of previous studies in which NPY was observed in neurons located within the SCG as an important neuronal factor taking part in the regulation of blood pressure, circadian rhythms and vasoconstriction. The results of previous studies show that NPY is especially important within the autonomic nervous system and the presence of this substance has been described in approximately two-thirds of neurons located in the sympathetic ganglia (Barczewska et al., 2017). The observations made in the present study indicate, for the first time, that NPY is also present in a relatively high percentage of neuronal cells supplying the oesophagus. This suggests that NPY is an important neuronal factor, not only within the enteric nervous system located in the wall of the GI tract (El-Salhy and Hausken, 2016; Tan et al., 2018), but also in the extrinsic innervation of the digestive tract. It should be noted that, contrary to other segments of the GI system (Mikołajczyk et al., 2017), knowledge about the distribution and functions of NPY in the oesophagus is relatively limited (Parkman et al., 1989). Although previous studies have reported the presence of NPY in neurons and nerves in the oesophageal wall (Rytel et al., 2018), the exact roles of this substance are still not fully elucidated. Probably they are similar to functions of NPY in other parts of the GI tract, where this substance is involved in the inhibition of intestinal motility and secretory activity, as well as the contraction of mesenteric blood vessels (Rosano et al., 2017). Moreover, some previous studies have also shown the participation of NPY in pathological processes within the GI tract (Gillard et al., 2016). Such functions may also appear in the oesophagus, as evidenced by changes in the distribution of oesophageal NPYpositive neurons during achalasia (Wattchow and Costa, 1996). In view of the fact that the majority of NPY-positive neurons supplying the oesophagus observed during the present study were also noradrenergic, it can be expected that in this part of the GI tract NPY displays the (above-mentioned) typical functions 

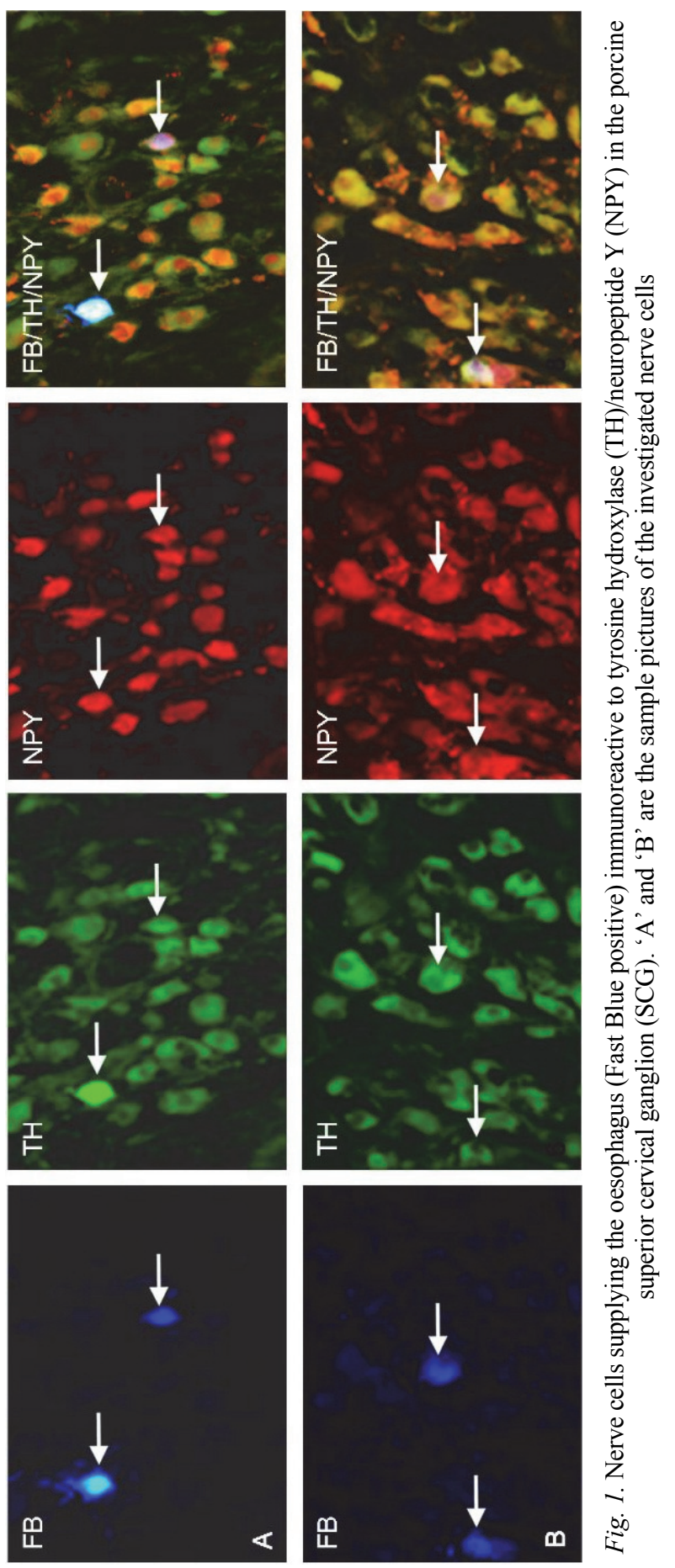
of sympathetic innervation. On the other hand, although the functions of a small neuronal population show the presence of NPY, TH negativity is completely unknown and requires further investigations. Nevertheless, the results obtained during the present study are an important step towards elucidating all aspects of the participation of NPY in the oesophageal innervation. Moreover, due to similarities in neurochemical organisation of the nervous systems between humans and the domestic pig (Gonzalez-Bulnes et al., 2016), this study may be the starting point of research aimed at expanding our knowledge on NPY-dependent mechanisms in the human oesophagus.

\section{References}

Barczewska, M., Juranek, J. and Wojtkiewicz, J. (2017): Origins and neurochemical characteristics of porcine intervertebral disc sympathetic innervation: a preliminary report. J. Mol. Neurosci. $63,50-57$.

Bedwell, S. A. and Tinsley, C. J. (2018): Mapping of fine-scale rat prefrontal cortex connections: Evidence for detailed ordering of inputs and outputs connecting the temporal cortex and sensory-motor regions. Eur. J. Neurosci. 48, 1944-1963.

Chandrasekharan, B., Bala, V., Kolachala, V. L., Vijay-Kumar, M., Jones, D., Gewirtz, A. T., Sitaraman, S. V. and Srinivasan, S. (2008): Targeted deletion of neuropeptide Y (NPY) modulates experimental colitis. PLoS One 3, e3304. doi: 10.1371/journal.pone.0003304

De Fontgalland, D., Wattchow, D. A., Costa, M. and Brookes, S. J. (2008): Immunohistochemical characterization of the innervation of human colonic mesenteric and submucosal blood vessels. Neurogastroenterol. Motil. 20, 1212-1226.

El-Salhy, M. and Hausken, T. (2016): The role of the neuropeptide Y (NPY) family in the pathophysiology of inflammatory bowel disease (IBD). Neuropeptides 55, 137-144.

Forbes, S. C. and Cox, H. M. (2014): Peptide YY, neuropeptide Y and corticotrophin-releasing factor modulate gastrointestinal motility and food intake during acute stress. Neurogastroenterol. Motil. 26, 1605-1614.

Gillard, L., Billiauws, L., Stan-Iuga, B., Ribeiro-Parenti, L., Jarry, A. C., Cavin, J. B., Cluzeaud, F., Mayeur, C., Thomas, M., Freund, J. N., Lacorte, J. M., Le Gall, M., Bado, A., Joly F. and Le Beyec, J. (2016): Enhanced ghrelin levels and hypothalamic orexigenic AgRP and NPY neuropeptide expression in models of jejuno-colonic short bowel syndrome. Sci. Rep. 6, 28345.

Gonkowski, S., Kaminska, B., Burlinski, P., Kroll, A. and Calka, J. (2009): The influence of drug resistant ulcerative colitis on the number of cocaine- and amphetamine-regulated transcript peptide-like immunoreactive (CART-LI) mucosal nerve fibres of the descending colon in children. Prz. Gastroenterol. 4, 147-151.

Gonzalez-Bulnes, A., Astiz, S., Ovilo, C., Lopez-Bote, C. J., Torres-Rovira, L., Barbero, A., Ayuso, M., Garcia-Contreras, C. and Vazquez-Gomez, M. (2016): Developmental origins of health and disease in swine: implications for animal production and biomedical research. Theriogenology 86, 110-119.

Grubišić, V., Verkhratsky, A., Zorec, R. and Parpura, V. (2018): Enteric glia regulate gut motility in health and disease. Brain Res. Bull. 136, 109-117.

Juranek, J. K. and Wojtkiewicz, J. A. (2015): Origins and neurochemical complexity of preganglionic neurons supplying the superior cervical ganglion in the domestic pig. J. Mol. Neurosci. 55, 297-304.

Konturek, S. J., Konturek, J. W., Pawlik, T. and Brzozowski, T. (2004): Brain-gut axis and its role in the control of food intake. J. Physiol. Pharmacol. 55, 137-154.

Landry, M., Holmberg, K., Zhang, X. and Hökfelt, T. (2000): Effect of axotomy on expression of NPY, galanin, and NPY Y1 and Y2 receptors in dorsal root ganglia and the superior cervi- 
cal ganglion studied with double-labeling in situ hybridization and immunohistochemistry. Exp. Neurol. 162, 361-384.

Louzao, M. C., Fernández, D. A., Abal, P., Fraga, M., Vilariño, N., Vieytes, M. R. and Botana, L. M. (2015): Diarrhetic effect of okadaic acid could be related with its neuronal action: Changes in neuropeptide Y. Toxicol. Lett. 237, 151-160.

Majewski, M., Bossowska, A., Gonkowski, S., Wojtkiewicz, J., Brouns, I., Scheuermann, D. W., Adriaensen, D. and Timmermans, J. P. (2002): Neither axotomy nor target-tissue inflammation changes the NOS- or VIP-synthesis rate in distal bowel-projecting neurons of the porcine inferior mesenteric ganglion (IMG). Folia Histochem. Cytobiol. 40, 151-152.

Malmström, R. E. (2000): Neuropeptide Y Y1 receptor mediated mesenteric vasoconstriction in the pig in vivo. Regul. Pept. 95, 59-63.

Masliukov, P. M., Konovalov, V. V., Emanuilov, A. I. and Nozdrachev, A. D. (2012): Development of neuropeptide Y-containing neurons in sympathetic ganglia of rats. Neuropeptides 46, 345-352.

Mikołajczyk, A., Gonkowski, S. and Złotkowska, D. (2017): Modulation of the main porcine enteric neuropeptides by a single low-dose of lipopolysaccharide (LPS) Salmonella Enteritidis. Gut Pathog. 9, 73.

Obremski, K., Gonkowski, S. and Wojtacha, P. (2015): Zearalenone-induced changes in the lymphoid tissue and mucosal nerve fibers in the porcine ileum. Pol. J. Vet. Sci. 18, 357-365.

Oltean, M., Jiga, L., Hellström, M., Söfteland, J., Papurica, M., Hoinoiu, T., Ionac, M. and Casselbrant, A. (2017): A sequential assessment of the preservation injury in porcine intestines. J. Surg. Res. 216, 149-157.

Parkman, H. P., Reynolds, J. C., Ogorek, C. P. and Kicsak, K. M. (1989): Neuropeptide Y augments adrenergic contractions at feline lower esophageal sphincter. Am. J. Physiol. 256, G589-597.

Rosano, G. M. C., Tousoulis, D., McFadden, E., Clarke, J., Davies, G. J. and Kaski, J. C. (2017): Effects of neuropeptide $\mathrm{Y}$ on coronary artery vasomotion in patients with microvascular angina. Int. J. Cardiol. 238, 123-127.

Rytel, L. and Calka, J. (2016): Acetylsalicylic acid-induced changes in the chemical coding of extrinsic sensory neurons supplying the prepyloric area of the porcine stomach. Neurosci. Lett. 617, 218-224.

Rytel, L., Szymanska, K., Gonkowski, I. and Wojtkiewicz, J. (2018): Neurochemical characterization of intramural nerve fibres in the porcine oesophagus. Anat. Histol. Embryol. 47, 517-526.

Sarnat, H. B. (2015): Immunocytochemical markers of neuronal maturation in human diagnostic neuropathology. Cell Tissue Res. 359, 279-294.

Szymanska, K., Makowska, K. and Gonkowski, S. (2018): The influence of high and low doses of bisphenol A (BPA) on the enteric nervous system of the porcine ileum. Int. J. Mol. Sci. 19, 917. pii: E917. doi: 10.3390/ijms 19030917

Tan, C. M. J., Green, P., Tapoulal, N., Lewandowski, A. J., Leeson, P. and Herring, N. (2018): The role of neuropeptide Y in cardiovascular health and disease. Front. Physiol. 9, 1281.

Wang, G. D., Wang, X. Y., Hu, H. Z., Fang, X. C., Liu, S., Gao, N. and Xia, Y. (2006): Plateletactivating factor in the enteric nervous system of the guinea pig small intestine. Am. J. Physiol. Gastrointest. Liver Physiol. 291, G928-937.

Wattchow, D. A. and Costa, M. (1996): Distribution of peptide-containing nerve fibres in achalasia of the oesophagus. J. Gastroenterol. Hepatol. 11, 478-485.

Wojtkiewicz, J., Juranek, J. K., Kowalski, I., Bladowski, M., Całka, J. and Majewski, M. (2011): Immunohistochemical characterization of superior cervical ganglion neurons supplying porcine parotid salivary gland. Neurosci. Lett. 500, 57-62.

Yu, X., Hu, Y., Ru, F., Kollarik, M., Undem, B. J. and Yu, S. (2015): TRPM8 function and expression in vagal sensory neurons and afferent nerves innervating guinea pig esophagus. Am. J. Physiol. Gastrointest. Liver Physiol. 308, G489-496.

Zalecki, M., Podlasz, P., Pidsudko, Z., Wojtkiewicz, J. and Kaleczyc, J. (2012): Vagal projections to the pylorus in the domestic pig (Sus scrofa domestica). Auton. Neurosci. 171, 21-27. 